\title{
A new GeV-TeV particle component and the barrier of cosmic-ray sea in the $\mathrm{CMZ}$ region
}

\author{
Xiaoyuan Huang, ${ }^{a, b, *}$ Qiang Yuan ${ }^{a, b}$ and Yi-Zhong Fan $^{a, b}$ \\ ${ }^{a}$ Key Laboratory of Dark Matter and Space Astronomy, Purple Mountain Observatory, Chinese Academy \\ of Sciences, Nanjing 210023, Chiny \\ ${ }^{b}$ School of Astronomy and Space Science, University of Science and Technology of China, Hefei, Anhui \\ 230026, China \\ E-mail: xyhuang@pmo.ac.cn, yuanq@pmo.ac.cn, yzfan@pmo.ac.cn
}

The Galactic center is widely anticipated to be an important cosmic-ray source and the observations of some Imaging Atmospheric Cherenkov Telescopes did successfully reveal a new component of $\mathrm{TeV}-\mathrm{PeV}$ cosmic rays in the vicinity of the Galactic center. This work reports the identification of $\mathrm{GeV}-\mathrm{TeV}$ cosmic rays in the central molecular zone with the $\gamma$-ray observations of the Fermi-LAT. The spectrum and spatial gradient of the GeV-TeV source component are consistent with that measured by the Imaging Atmospheric Cherenkov Telescopes. The inferred cosmic-ray energy density in the central molecular zone region is, however, substantially lower than the so-called cosmic-ray sea component. Our finding is in support of the presence of a high energy particle accelerator at the Galactic center and strongly suggests a barrier that can effectively suppress the penetration of the particles from the cosmic-ray sea to the central molecular zone.

$37^{\text {th }}$ International Cosmic Ray Conference (ICRC 2021)

July 12 th - 23rd, 2021

Online - Berlin, Germany

\footnotetext{
${ }^{*}$ Presenter
} 


\section{Introduction}

It is believed that in the Milky Way cosmic rays (CRs) could be accelerated by shocks in supernova remnants [1, and references therein] or massive star wind [2]. Those charged relativistic particles would then propagate diffusively in the Galactic magnetic field, possibly experiencing reacceleration, convection, spallation, and energy loss processes [3, 4]. This production and propagation process would lead to a large-scale, quasi-steady-state CR sea, which distributes relatively smoothly in the Galaxy, as supported by the Fermi-LAT observations [5-7].

However, in the proximity of a recent or currently active accelerator, the smoothly distributed CR sea would be overlaid with a new component of fresh CRs. Observations of such a fresh CR component will be very important in studying the acceleration, injection, and transportation processes of CRs. In the central molecular zone (CMZ) region, the High Energy Stereoscopic System (H.E.S.S.) and other Imaging Atmospheric Cherenkov Telescopes (IACTs) reveal a large amount of very-high-energy (VHE) CRs with a hard spectrum and a high density (a factor of $\sim 10$ times higher than that measured locally at the Earth), implying an injection of CRs by a source close to the GC [8-10]. Further studies [11-13] show that there is a gradient of the CR density profile, $\propto r^{-\alpha}(\alpha \sim 1-1.2)$, deduced from the $\gamma$-ray profile deconvolved the gas density in the CMZ. One attractive explanation is that Sagittarius $A^{\star}$, which might have been more active in the past, accelerated CRs up to $\mathrm{PeV}$ energies which diffuse outwards and collide with molecular gas to produce energetic $\gamma$-rays.

The search for a possible counterpart of the VHE component in the low-energy $\gamma$-ray band would be very important in revealing its nature $[14,15]$. In the analysis, the so-called Galactic Center $\mathrm{GeV}$ excess [GCE; e.g., 16] that could be either from dark matter annihilation or a group of unresolved faint point sources like millisecond pulsars [17] may play an important role. Though this excess peaks at several $\mathrm{GeV}$, at several tens of $\mathrm{GeV}$ it may be still bright enough to contaminate other emission signals $[18,19]$. A dedicated morphological and spectral analysis of the Fermi-LAT data in the GC region, with proper consideration of the GCE component, is thus necessary to critically address the $\mathrm{CR}$ population in the most central region.

\section{The setup of the Fermi-LAT data analysis}

The Fermi-LAT data ${ }^{1}$ of version P8R3 and class SOURCE are used in this analysis. We select the data recorded from August 4, 2008 to February 1, 2020. We select photons with energies higher than $8 \mathrm{GeV}$, which have a balance of a good angular resolution and a large enough statistics. To suppress the contamination from $\gamma$-ray generated by CR interactions in the upper atmosphere, photons collected at zenith angles larger than $90^{\circ}$ are removed. Moreover, we adopt the specifications (DATA_QUAL $>0$ ) \& \& (LAT_CONFIG==1) to select good quality data. We bin the data, from 8 $\mathrm{GeV}$ to $500 \mathrm{GeV}$, into 20 logarithmically distributed energy bins and $100 \times 100$ spatial bins with pixel size of $0.1^{\circ}$ centered at the GC. We employ the binned likelihood analysis method to analyze the data with the Fermitools version 1.2.1. The instrument response function (IRF) adopted is P8R3_SOURCE_V2.

\footnotetext{
${ }^{1}$ https://fermi.gsfc.nasa.gov/ssc/data/access/
} 
The source model XML file is generated using the user contributed tool make4FGLxml. py ${ }^{2}$ based on the 4FGL catalog [20,21]. The spectrum of Sagittarius $A^{\star}$ is modeled as a power-law instead of the default log-parabola spectrum. Two additional point sources, 3FHL J1747.2-2822 and 3FHL J1748.6-2816 which are not in the 4FGL source catalog gll_psc_v23. xml ${ }^{3}$ but in the 3FHL source catalog gll_psch_v12. $\mathrm{xml}^{4}$, are also added in our analysis.

We use the Galactic diffusion emission (GDE) modeled by the GALPROP software ${ }^{5}$ [22, 23] in this work, which gives different components of the diffuse emission individually. The result of model A as introduced in [24] is taken as our baseline GDE model. For systematics studies about the template tracing neutral pion decay, besides the mentioned GALPROP generated model, we also employ the Planck dust opacity map ${ }^{6}$ from [25] as an alternative, which traces the gas distributions without the uncertainty from the $\mathrm{X}_{C O}$ factor in the GALPROP related templates. The bremsstrahlung in our energy range is expected to be small and is thus neglected. The $\gamma$-ray emissions from the inverse Compton scattering of high energy electrons off the optical, infrared, and the cosmic microwave background are taken as another template. The third diffuse template is the isotropic background. Power-law spectra of these three diffuse templates are assumed, and both the normalizations and spectral indices are treated as free parameters in the likelihood fit. For the GCE component, we use a line-of-sight integration of $\rho^{2}(r)$, where $\rho(r) \propto r^{-1.28}$ as derived in [19], to model its spatial distribution, and again assume a power-law to model its spectrum. As shown in [19], the power-law spectrum is reasonable to describe the high-energy tail of the GCE.

\section{CRs with low density in the CMZ}

We single out the $\mathrm{CMZ}$ region [26] for studying CR properties near the GC region, and the rest is referred to as off-CMZ region. The spectral indices and normalizations of the $\mathrm{CMZ}$ and off-CMZ regions are independent in the fitting. The spectral index of the CMZ region is harder by $0.19 \pm 0.07$ than that, about 2.68, predicted in the GALPROP GDE model A, and the integrated energy flux is $(7.05 \pm 0.44) \times 10^{-5} \mathrm{MeV} \mathrm{cm}^{-2} \mathrm{~s}^{-1}$ from $8 \mathrm{GeV}$ to $500 \mathrm{GeV}$. We find that the spectral index of the CMZ is consistent with that observed in the VHE $\gamma$-ray band $\left(2.32 \pm 0.05_{\text {stat }} \pm 0.10_{\text {syst }}\right.$ and $2.28 \pm 0.03_{\text {stat }} \pm 0.20_{\text {syst }}$ by H.E.S.S. [11-13]), but is clearly harder than that expected from the CR sea interaction with the gas in the CMZ. Furthermore, the integrated flux is lower than that predicted from the CR sea interaction. Similar results have been obtained if we change the GALPROP template to the Planck template. Our results thus imply that there is a hard component in the CMZ which possibly coincides with the VHE component, and the CR density of the background sea component in the $\mathrm{CMZ}$ region should be suppressed.

Then we investigate the CR density distribution in the CMZ and off-CMZ directions. To enable a high enough spatial resolution of the gas distribution, we use the Planck dust opacity map as a tracer of the line-of-sight integral of the gas distribution. We split the Planck map into small segments, as shown in the left panel of Figure 1, with a width of 0.5 degrees except for the last one.

\footnotetext{
${ }^{2} \mathrm{http} / / /$ fermi.gsfc.nasa.gov/ssc/data/analysis/user/

${ }^{3} \mathrm{https} / / /$ fermi.gsfc.nasa.gov/ssc/data/access/lat/10yr_catalog/

${ }^{4} \mathrm{https}: / /$ fermi.gsfc.nasa.gov/ssc/data/access/lat/3FHL/

${ }^{5} \mathrm{http}: / /$ galprop.stanford.edu/

${ }^{6} \mathrm{https} / /$ irsa.ipac.caltech.edu/data/Planck/release_2/all-sky-maps/maps/componentmaps/foregrounds/COM_CompMap_DustGNILC-Model-Opacity_2048_R2.01.fits
} 
We model each segment with a power-law spectrum by fixing the spectral index, to be those derived above using the Planck template, which are 2.56 in the CMZ region, and 2.64 in the off-CMZ region. We fix all the parameters of point sources outside the $\mathrm{CMZ}$ to their fitting values as well and leave all other source parameters free. The CR densities $\left(w_{\mathrm{CR}}\right.$ in $\left.\mathrm{eV} / \mathrm{cm}^{3}\right)$ in those segments can thus be estimated from the normalizations (and hence luminosities) of the $\gamma$-ray emission as

$$
\begin{aligned}
w_{\mathrm{CR}}\left(E_{\mathrm{CR}}\right) & \approx 0.018\left(\frac{\eta_{N}}{1.5}\right)^{-1} \\
& \times\left(\frac{L_{\gamma}\left(E_{\gamma}\right)}{10^{34} \mathrm{erg} / \mathrm{s}}\right)\left(\frac{M}{10^{6} M_{\odot}}\right)^{-1},
\end{aligned}
$$

where $E_{\mathrm{CR}} \approx 10 E_{\gamma}$ is the corresponding energy of CRs giving $\gamma$-ray energy of $E_{\gamma}, \eta_{N}$ accounts for the correction from nuclei heavier than protons and is taken as 1.5 in this work, $L_{\gamma}\left(E_{\gamma}\right)$ is the $\gamma$-ray luminosity and $M$ is the total mass of the gas in the segment which is estimated using the relation between the dust opacity and the column density for the Planck dust opacity map [27]. Using parameters for each segment derived in the fitting, the distribution of $w_{\mathrm{CR}}(80 \mathrm{GeV} \leq E \leq 5 \mathrm{TeV})$ is shown in the right panel of Figure 1. It is very interesting to find that, for segments outside the $\mathrm{CMZ}$, the CR density is almost a constant, which is consistent with the expectation of the CR sea in such a small region. The CR density in the CMZ, however, declines clearly with the distance to the GC, which is consistent with a gradient profile observed at the VHE band. The CR density in the CMZ is also generally smaller than that outside the CMZ.
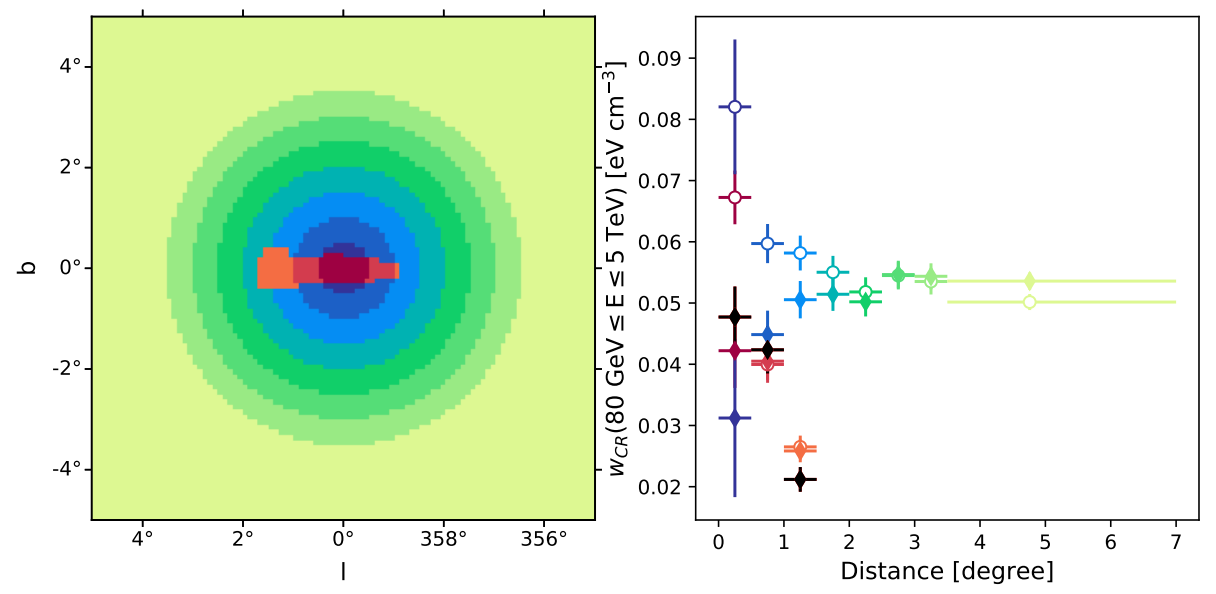

Figure 1: Left: The segment division of the GC region for the Planck map. Segments in the CMZ are marked in red to orange and segments in the off-CMZ region are marked in green to blue. Right: CR densities in different segments of the Planck map defined in the left panel, with same colors. Filled and open symbols represent results from fittings with and without the GCE, respectively. CR densities, inferred from analysis with the CS map, for segments within the CMZ are marked with black points, and they agree well with those derived from fitting with the Planck map. 


\section{The GeV-TeV counterpart of the new CR component discovered by the IACTs}

The VHE observations of the new component in the GC show a power-law radial profile, $\propto r^{-\alpha}$, of the CR density with $\alpha=1.10 \pm 0.12$ [11] and $1.2 \pm 0.3$ [13]. However, in the GALPROP GDE model the spatial gradient of CR densities in the very center region of the Galaxy is not properly modeled. Furthermore, the molecular hydrogen component used in GALPROP is traced with the $\mathrm{CO}$ emission, which may be strongly contaminated by the foreground and background emissions in the GC. The CS radio map [26] is expected to be a better tracer of the molecular component $[11,13]$. Therefore we use a power-law function $r^{-\alpha}$ to parameterize the radial distribution of the CR density in the CMZ, and multiply its line-of-sight integral with the CS emission template to model the spatial distribution of the new component. The spatial template of the off-CMZ region is adopted as the GALPROP template. We scan $\alpha$ from 0.0 to 2.0 and we find that the Fermi-LAT data favors a non-zero value of $\alpha$ at a confidence level of $5.4 \sigma$. The fitting result is $\alpha=1.35_{-0.09}^{+0.06}$, which is consistent with that derived in the VHE band, $\alpha=1.2 \pm 0.3$ in [13] and $\alpha=1.1 \pm 0.12$ in [11].To visualize the radial dependence of the CR density and to investigate the dependence on the mass estimation with different observations, we derive the CR density in the CMZ region using the CS map without radial scaling divided into 3 segments again. We estimate the mass for each segments, using the relation between the total CS fluxes and the mass. As shown in the right panel of Figure 1, the CR densities in the 3 segments within the CMZ, inferred from the CS map, agree well those inferred from the Plank map.

We derive the spectral energy distributions (SED) of the new component in the CMZ region, using the CS $\times r^{-1.35}$ template, and show the results in Figure 2. The $\gamma$-ray spectral index of the fitting in the whole energy band from $8 \mathrm{GeV}$ to $500 \mathrm{GeV}$ is $2.50 \pm 0.08$, which is roughly consistent with that measured in the VHE band $\left(2.32 \pm 0.05_{\text {stat }} \pm 0.10_{\text {syst }}\right.$ and $2.28 \pm 0.03_{\text {stat }} \pm 0.20_{\text {syst }}$ by H.E.S.S. $[11,12])$. The $\gamma$-ray flux in the inner $150 \mathrm{pc}\left( \pm 1^{\circ}\right)$ region is $(5.50 \pm 0.38) \times 10^{-5}$ $\mathrm{MeV} \mathrm{cm} \mathrm{s}^{-2}$. Taking the mass of dense molecular clouds as $3 \times 10^{7} \mathrm{M}_{\odot}[26,28]$ and $\eta_{N}=1.5$, we obtain the CR density in the $\mathrm{CMZ}$ as $(4.5 \pm 0.3) \times 10^{-2} \mathrm{eV} \mathrm{cm}^{-3}$ from $80 \mathrm{GeV}$ to $5 \mathrm{TeV}$, which is close to the local CR density, about $4.2 \times 10^{-2} \mathrm{eV} \mathrm{cm}^{-3}$ at this energy range [29, 30]. And the proton density above $10 \mathrm{GeV},(4.3 \pm 0.3) \times 10^{-12} \mathrm{~cm}^{-3}$ if the power-law spectrum still holds for energy down to $10 \mathrm{GeV}$, is a factor of a few smaller than the expectation, about $12 \times 10^{-12} \mathrm{~cm}^{-3}$, of the $\mathrm{CR}$ sea in the GC region [31]. We also note that the derived proton density in the CMZ region in [31] is also lower than that from the prediction, and this supports our results.

\section{Conclusions}

Here, we report the re-analysis of the Fermi-LAT data in the CMZ region and the identification of a component of GeV-TeV CRs, which is likely the counterpart of the new TeV-PeV CR component discovered by the IACTs. This supports the presence of a high-energy particle accelerator at the GC. Unexpectedly, we further show that the inferred energy density from the background CRs in the CMZ region is clearly smaller than that from an extrapolation of the CR sea distribution. A natural explanation is that there is a barrier in the $\mathrm{CMZ}$, maybe due to the strong magnetic field in such a region, that can effectively suppress the penetration of particles from outside to the CMZ. 


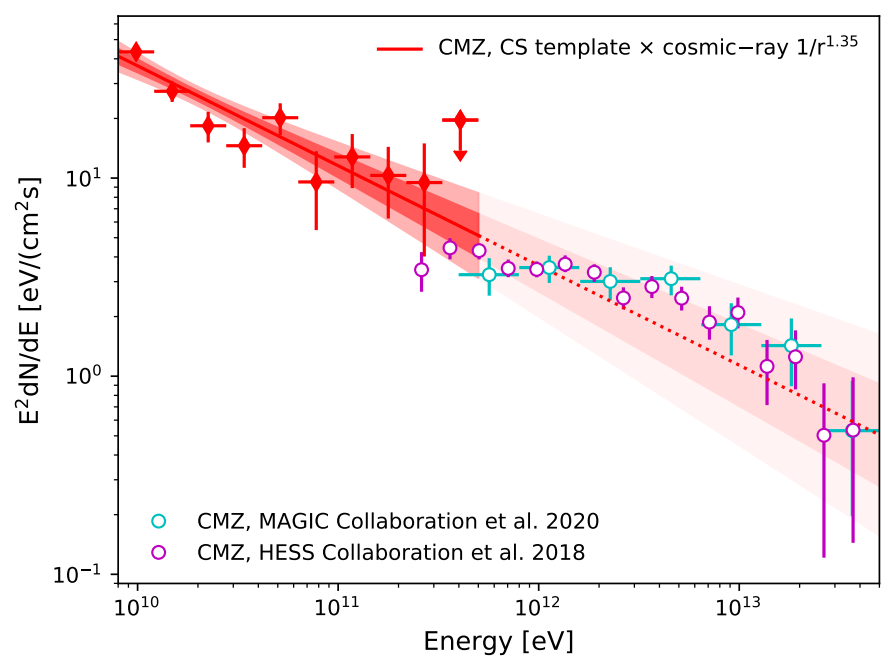

Figure 2: SED of CMZ component obtained using the Fermi-LAT data (red filled dots), compared with those measured by H.E.S.S. [11] and MAGIC [13]. The solid lines and associated bands show the best-fit power-law model and the $68 \%$ and $95 \%$ confidence ranges.

\section{References}

[1] P. Blasi, The origin of galactic cosmic rays, Astron. Astrophys. Rev. 21 (2013) 70 [1311.7346].

[2] F. Aharonian, R. Yang and E. de Oña Wilhelmi, Massive stars as major factories of Galactic cosmic rays, Nature Astronomy 3 (2019) 561 [1804.02331].

[3] T.K. Gaisser, Cosmic rays and particle physics., Cambridge University Press (1990).

[4] A.W. Strong, I.V. Moskalenko and V.S. Ptuskin, Cosmic-Ray Propagation and Interactions in the Galaxy, Annual Review of Nuclear and Particle Science 57 (2007) 285 [astro-ph/0701517].

[5] R.-z. Yang, D.I. Jones and F. Aharonian, Fermi-LAT observations of the Sagittarius B complex, Astron. Astrophys. 580 (2015) A90 [1410. 7639].

[6] Z.-Q. Shen, X. Huang, Q. Yuan, Y.-Z. Fan and D.-M. Wei, Probing local cosmic rays using Fermi-LAT observations of a mid-latitude region in the third Galactic quadrant, Phys. Rev. D 99 (2019) 123001 [1904.12264].

[7] F. Aharonian, G. Peron, R. Yang, S. Casanova and R. Zanin, Probing the sea of galactic cosmic rays with Fermi-LAT, Phys. Rev. D 101 (2020) 083018 [1811.12118].

[8] F. Aharonian, A.G. Akhperjanian, A.R. Bazer-Bachi, M. Beilicke, W. Benbow, D. Berge et al., Discovery of very-high-energy $\gamma$-rays from the Galactic Centre ridge, Nature 439 (2006) 695 [astro-ph/0603021]. 
[9] A. Archer, W. Benbow, R. Bird, M. Buchovecky, J.H. Buckley, V. Bugaev et al., TeV Gamma-Ray Observations of the Galactic Center Ridge by VERITAS, Astrophys. J. 821 (2016) 129 [1602.08522].

[10] M.L. Ahnen, S. Ansoldi, L.A. Antonelli, P. Antoranz, C. Arcaro, A. Babic et al., Observations of Sagittarius A* during the pericenter passage of the G2 object with MAGIC, Astron. Astrophys. 601 (2017) A33 [1611.07095].

[11] H. E. S. S. Collaboration, A. Abramowski, F. Aharonian, F.A. Benkhali, A.G. Akhperjanian, E.O. Angüner et al., Acceleration of petaelectronvolt protons in the Galactic Centre, Nature 531 (2016) 476 [1603.07730].

[12] H. E. S. S. Collaboration, H. Abdalla, A. Abramowski, F. Aharonian, F. Ait Benkhali, A.G. Akhperjanian et al., Characterising the VHE diffuse emission in the central 200 parsecs of our Galaxy with H.E.S.S., Astron. Astrophys. 612 (2018) A9 [1706. 04535].

[13] MAGIC Collaboration, V.A. Acciari, S. Ansoldi, L.A. Antonelli, A. Arbet Engels, D. Baack et al., MAGIC observations of the diffuse $\gamma$-ray emission in the vicinity of the Galactic Centre, arXiv e-prints (2020) arXiv:2006.00623 [2006.00623].

[14] O. Macias and C. Gordon, Contribution of cosmic rays interacting with molecular clouds to the Galactic Center gamma-ray excess, Phys. Rev. D 89 (2014) 063515 [1312.6671].

[15] D. Gaggero, D. Grasso, A. Marinelli, M. Taoso and A. Urbano, Diffuse Cosmic Rays Shining in the Galactic Center: A Novel Interpretation of H.E.S.S. and Fermi-LAT $\gamma$-Ray Data, Phys. Rev. Lett. 119 (2017) 031101 [1702.01124].

[16] D. Hooper and L. Goodenough, Dark matter annihilation in the Galactic Center as seen by the Fermi Gamma Ray Space Telescope, Physics Letters B 697 (2011) 412 [1010 . 2752].

[17] L.J. Chang, S. Mishra-Sharma, M. Lisanti, M. Buschmann, N.L. Rodd and B.R. Safdi, Characterizing the nature of the unresolved point sources in the Galactic Center: An assessment of systematic uncertainties, Phys. Rev. D 101 (2020) 023014 [1908. 10874].

[18] B. Zhou, Y.-F. Liang, X. Huang, X. Li, Y.-Z. Fan, L. Feng et al., GeV excess in the Milky Way: The role of diffuse galactic gamma-ray emission templates, Phys. Rev. D 91 (2015) 123010 [1406.6948].

[19] F. Calore, I. Cholis and C. Weniger, Background model systematics for the Fermi GeV excess, J. Cosmol. Astropart. Phys. 2015 (2015) 038 [1409.0042].

[20] S. Abdollahi, F. Acero, M. Ackermann, M. Ajello, W.B. Atwood, M. Axelsson et al., Fermi Large Area Telescope Fourth Source Catalog, Astrophys. J. Supp. 247 (2020) 33 [1902.10045].

[21] J. Ballet, T.H. Burnett, S.W. Digel and B. Lott, Fermi Large Area Telescope Fourth Source Catalog Data Release 2, arXiv e-prints (2020) arXiv:2005.11208 [2005 . 11208]. 
[22] A.W. Strong, I.V. Moskalenko and O. Reimer, Diffuse Continuum Gamma Rays from the Galaxy, Astrophys. J. 537 (2000) 763 [astro-ph/9811296].

[23] A.E. Vladimirov, S.W. Digel, G. Jóhannesson, P.F. Michelson, I.V. Moskalenko, P.L. Nolan et al., GALPROP WebRun: An internet-based service for calculating galactic cosmic ray propagation and associated photon emissions, Computer Physics Communications 182 (2011) 1156 [1008.3642].

[24] M. Ackermann, M. Ajello, A. Albert, W.B. Atwood, L. Baldini, J. Ballet et al., The Spectrum of Isotropic Diffuse Gamma-Ray Emission between $100 \mathrm{MeV}$ and $820 \mathrm{GeV}$, Astrophys. J. 799 (2015) 86 [1410 . 3696].

[25] Planck Collaboration, N. Aghanim, M. Ashdown, J. Aumont, C. Baccigalupi, M. Ballardini et al., Planck intermediate results. XLVIII. Disentangling Galactic dust emission and cosmic infrared background anisotropies, Astron. Astrophys. 596 (2016) A109 [1605. 09387].

[26] M. Tsuboi, T. Handa and N. Ukita, Dense Molecular Clouds in the Galactic Center Region. I. Observations and Data, Astrophys. J. Supp. 120 (1999) 1.

[27] PLANCK collaboration, Planck Early Results: All sky temperature and dust optical depth from Planck and IRAS: Constraints on the 'dark gas' in our galaxy, Astron. Astrophys. 536 (2011) A19 [1101.2029].

[28] K. Ferrière, W. Gillard and P. Jean, Spatial distribution of interstellar gas in the innermost 3 kpc of our galaxy, Astron. Astrophys. 467 (2007) 611 [astro-ph/0702532].

[29] AMS collaboration, Precision Measurement of the Proton Flux in Primary Cosmic Rays from Rigidity 1 GV to 1.8 TV with the Alpha Magnetic Spectrometer on the International Space Station, Phys. Rev. Lett. 114 (2015) 171103.

[30] DAMPE collaboration, Measurement of the cosmic-ray proton spectrum from $40 \mathrm{GeV}$ to 100 TeV with the DAMPE satellite, Sci. Adv. 5 (2019) eaax3793 [1909. 12860].

[31] F. Acero, M. Ackermann, M. Ajello, A. Albert, L. Baldini, J. Ballet et al., Development of the Model of Galactic Interstellar Emission for Standard Point-source Analysis of Fermi Large Area Telescope Data, Astrophys. J. Supp. 223 (2016) 26 [1602 . 07246]. 